\title{
Statistical description of the denatured structure of a single protein, staphylococcal nuclease, by FRET analysis
}

\author{
Mariko Yamaguchi ${ }^{1,2} \cdot$ Emi Ohta $^{1} \cdot$ Takuya Muto $^{1} \cdot$ Takayoshi Watanabe $^{3} \cdot$ \\ Takahiro Hohsaka $^{3}$ - Yoichi Yamazaki ${ }^{1}$ - Hironari Kamikubo ${ }^{1}$ • Mikio Kataoka ${ }^{1,4}$ (ID
}

Received: 24 October 2017 / Accepted: 7 November 2017 / Published online: 25 November 2017

(C) The Author(s) 2017. This article is an open access publication

\begin{abstract}
Structural characterization of fully unfolded proteins is essential for understanding not only protein-folding mechanisms, but also the structures of intrinsically disordered proteins. Because an unfolded protein can assume all possible conformations, statistical descriptions of its structure are most appropriate. For this purpose, we applied Förster resonance energy transfer (FRET) analysis to fully unfolded staphylococcal nuclease. Artificial amino acids labeled with a FRET donor or acceptor were introduced by an amber codon and a four-base codon respectively. Eight double-labeled proteins were prepared, purified, and subjected to FRET analysis in $6 \mathrm{M}$ urea. The observed behavior could be explained by a power law, $R=\alpha N^{0.44}$, where $R$, and $N$ are the distance and the number of residues between donor and acceptor, and $\alpha$ is a coefficient. The index was smaller than the value expected for an excluded-volume random coil, 0.588 , indicating that the fully unfolded proteins were more compact than polypeptides in good solvent. The FRET efficiency in the native state did not necessarily correlate to the distance obtained from crystal structure, suggesting that other factors such as the orientation factor made a substantial contribution to FRET.
\end{abstract}

Keywords Protein $\cdot$ Denatured structure $\cdot$ FRET $\cdot$ Random coil $\cdot$ Staphylococcal nuclease

\section{Introduction}

Protein stability and folding are among the most important topics in protein science. Many excellent studies have been published on this subject, including the pioneering work of Anfinsen, who was awarded the Nobel Prize in Chemistry

Takuya Muto died of leukemia during the study. His experimental contribution was essential for this study.

This article is part of a Special Issue on 'Biomolecules to Bio-nanomachines — Fumio Arisaka 70th Birthday’ edited by Damien Hall, Junichi Takagi and Haruki Nakamura.

Mikio Kataoka

m_kataoka@cross.or.jp

1 Graduate School of Materials Science, Nara Institute of Science and Technology, Ikoma, Nara 630-0192, Japan

2 Present address: IMRA America, Inc., 48834 Kato Road, Fremont, CA 94538, USA

3 School of Materials Science, Japan Advanced Institute of Science and Technology, Nomi, Ishikawa 923-1292, Japan

4 Present address: Research Center for Neutron Science and Technology, Comprehensive Research Organization for Science and Society (CROSS), 162-1 Shirakata, Tokai, Ibaraki 319-1106, Japan
1972 (Anfinsen 1972). The folded structure of a protein is located at the minimum of the free-energy potential surface. Hence, folding is considered as a potential-minimum search process starting at a high-energy state (Levinthal 1968; Dill 1993; Onuchic et al. 1995; Dill and Chan 1997). Recent advances in structural and computational biology have provided deep insight into the stabilities and folding mechanisms of proteins. The atomic structures of various proteins have been solved to understand necessary atomic interaction for folding. The solved structure is the destination of folding. On the other hand, detailed structural information about denatured or unfolded structures, the initial point of folding, remains insufficient.

The importance of information on denatured structures began to be emphasized in the 1990's (Dill and Shortle 1991). The denatured structure is considered as the initial state in the process of protein-folding. Exploration of the folding mechanism by simulation is based on the idea that the initial state can assume all possible conformations, i.e., that it is a random coil (Pappu et al. 2000), but it remains to be resolved whether this is truly the case. On one hand, the scaling of the hydrodynamic radius as a function of molecular weight (chain length) indicates that the denatured state exhibits random coil behavior (Tanford et al. 1966; Kohn et al. 2004). On the other hand, NMR studies have shown that several residual structures remain in the denatured state 
(Neri et al. 1992; Kazmirski et al. 2001; Shortle and Ackerman 2001), and mutations affect both denatured and folded structures (Shortle and Meeker 1986); together, these observations suggest that the denatured structure is not necessarily a random coil. The apparent discrepancy may arise from the fact that the former studies were based on a statistical description of various proteins, whereas the latter were based on local structural information for a single protein. Accordingly, a statistical description of the structure of a single protein molecule in the denatured state would reconcile the discrepancy. To this end, we investigated the pairwise distances between residues over the entire polypeptide chain in a single protein, staphylococcal nuclease (SNase), using Förster resonance energy transfer (FRET).

To conduct FRET structure analysis, we need to introduce both donor and acceptor fluorescent dyes into a protein. For this purpose, a standard method is labeling of a reactive residue, such as cysteine or lysine. However, specific double-labeling at the desired positions and purification of properly labeled proteins are challenging, and it is not straightforward to prepare several sets of proteins double-labeled at different positions. To overcome these difficulties, we applied a labeling method that uses the amber codon and an artificial four-base codon (Hohsaka et al. 1999), corresponding to the donor BODIPY FL amino phenylalanine and acceptor BODIPY 558 amino phenylalanine (Kajihara et al. 2006) respectively. The donor site is designated by the amber codon, and the acceptor site is fixed at the fifth amino acid position by the four-base codon, CGGG. At the C-terminal end, the 6histidine tag is introduced to facilitate purification. A total of eight double-labeled proteins were prepared and purified. The donor site of each protein was located at residue $33,48,70,97,123,134,143$, or 146; accordingly, the double-labeled proteins are represented as, e.g., $33_{\mathrm{D}} 5_{\mathrm{A}}$ and $48_{\mathrm{D}} 5_{\mathrm{A}}$.

Measurement of fluorescence spectra and derivation of FRET efficiency were successful for all labeled SNases with and without $6 \mathrm{M}$ urea. The influence of urea on the fluorescence of donor and acceptor dyes was negligible compared to the FRET between the dyes introduced into a single SNase molecule. Complete unfolding of SNase at $6 \mathrm{M}$ urea was confirmed by $\mathrm{CD}$ spectra. The FRET efficiency at $6 \mathrm{M}$ urea showed that the $R-N$ relationship obeys a power law with an index of 0.44 , where $R$ and $N$ are the distance and the number of residues between donor and acceptor. This is smaller than the index of an excluded-volume random coil, 0.588 (De Gennes 1979; Kohn et al. 2004). The statistical description of the unfolded structure of a single protein suggests that the unfolded structure cannot be described by well-known random-coil polymer statistics, but instead is more compact. This is the first statistical description of the denatured structure of a single protein. Our findings indicate that FRET analysis of a single polypeptide is both effective and promising.

\section{Materials and methods}

\section{Design of DNA sequence}

Figure 1 shows the DNA sequence for the protein with the acceptor site at the fifth position and the donor site at the 33rd position $\left(33_{\mathrm{D}} 5_{\mathrm{A}}\right)$. When the four-base codon, CGGG, is translated into CGG as a normal three-base codon, a frameshift occurs, and a polypeptide with a completely different amino acid sequence is generated. To avoid such misreading, the expected stop codon (TAA) appears immediately. This strategy was also used for the other seven polypeptides. The donor site is designated by the amber codon, TAG. At the C-terminal end, the 6-histidine tag is added for purification. To avoid introducing another donor, the stop codon used in this construct is TGA instead of TAG. Only the successfully translated polypeptide can be purified on Ni-chelate beads. Two singlelabeled proteins with either the four-base codon or the amber codon at the fifth position $\left(5_{\mathrm{D}}\right.$ or $5_{\mathrm{A}}$ respectively) were also prepared.

\section{Protein expression and purification}

Aminoacyl tRNA of BODIPY-phenylalanine with the anticodon for the four-base codon or amber codon was synthesized in each experimental run using a previously described chemical aminoacylation method (Kajihara et al. 2006; Iijima and Hohsaka 2009). Proteins were expressed using a cell-free expression system containing the aminoacyl tRNA and relevant mRNA, as previously described (Iijima and Hohsaka 2009). The volume of each cell-free reaction was $100 \mu$ l. Full-length double-labeled proteins were purified on NiNTA beads (MagneHis Ni-particles, Promega, Madison, WI, USA) and desalted using a Zeba desalting column (Thermo Fisher Scientific, Waltham, MA, USA). The proteins were eluted in fluorescence measurement buffer ( $30 \mathrm{mM}$ sodium phosphate, $300 \mathrm{mM} \mathrm{NaCl}, 0.005 \%$ Brij-35, 0.1\% PEG-8000, $\mathrm{pH}$ 7.8).

Expression and purification were monitored by fluorescence imaging of PAGE gels and western blotting. The gel pattern was recorded on an LAS4000 (GE Healthcare) and an FMBIO-III (Hitachi Software Engineering).

\section{Measurement of fluorescence}

Fluorescence spectra were measured on an F-2500 fluorescence spectrophotometer (Hitachi) immediately after purification to prevent chromophores from degrading. After preparation of a $100 \mu \mathrm{l}$ solution either with or without $6 \mathrm{M}$ urea, two fluorescence spectra were measured with sequential excitation at $490 \mathrm{~nm}$ and $530 \mathrm{~nm}$. The ratio of the fluorescence intensity at $570 \mathrm{~nm}$ to that at $490 \mathrm{~nm}, R I$, was obtained from the fluorescence spectra with $490 \mathrm{~nm}$ excitation for further analysis. 
Fig. 1 Designed DNA sequence for the acceptor site at the fifth position and the donor site at the 33 rd position $\left(33_{\mathrm{D}} 5_{\mathrm{A}}\right)$. The fourbase codon and amber codon are demarcated by a solid box and a broken box respectively. The single underline represents the expected stop codon when the four-base codon is read as an ordinary three-base codon and a frameshift occurs. The double underline represents the 6xHistag. The stop codon, indicated by the wavy underline, is TGA

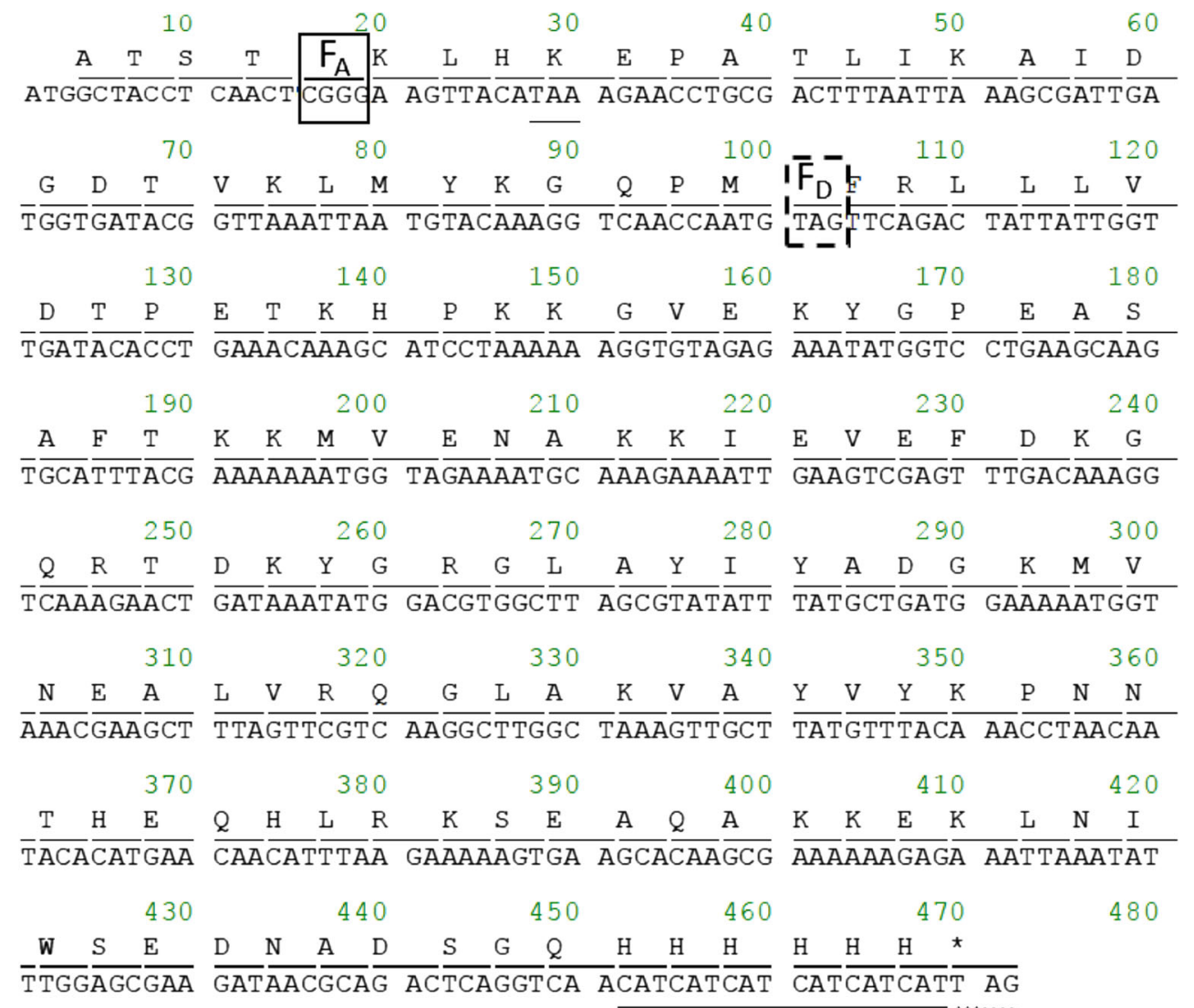

\section{Analysis of fluorescence spectra}

FRET efficiency, $E$, is expressed as

$E=\frac{1}{1+\left(\frac{R}{R_{0}}\right)^{6}}$.

$R_{0}$ is the Förster distance, at which the FRET efficiency is 0.5 , expressed as

$R_{0}^{6}=\frac{9 Q_{0}(\ln 10) \kappa^{2} J}{128 \pi^{5} n^{4} N_{A}}$,

where $Q_{0}, J, \kappa^{2}, n$, and $N_{\mathrm{A}}$ are the quantum yield of donor, the overlap integral of the fluorescence spectrum of the donor and the absorption spectrum of the acceptor, the orientation factor, the refractive index of medium, and Avogadro's number respectively (Valeur 2001). In the present case, the Förster distance was estimated to be $59.6 \AA$ (Iijima and Hohsaka 2009).

Experimentally, $E$ is estimated from $R I$. To derive the relationship between $E$ and $R I$, we first define the fluorescence spectrum of a double-labeled protein $I_{\mathrm{DA}}$ as follows:

$I_{\mathrm{DA}}=C_{\mathrm{D}} I_{\mathrm{D}}+C_{\mathrm{A}} I_{\mathrm{A}}$

where $I_{\mathrm{D}}$ and $I_{\mathrm{A}}$ are the normalized fluorescence spectra of donor and acceptor respectively. The fluorescence spectrum of a single-labeled protein, $5_{\mathrm{D}}\left(5_{\mathrm{A}}\right)$, was used to obtain $I_{\mathrm{D}}\left(I_{\mathrm{A}}\right)$. $C_{\mathrm{D}}$ and $C_{\mathrm{A}}$ are the fluorescence intensities of donor and acceptor respectively. From Eq. (3), RI can be expressed in terms of $C_{\mathrm{D}}$ and $C_{\mathrm{A}}$ as

$R I=\frac{C_{\mathrm{A}}}{C_{\mathrm{D}}}$.

$C_{\mathrm{A}}$ and $C_{\mathrm{D}}$ are defined by Eq. (5):

$C_{\mathrm{A}}=\varepsilon_{\mathrm{D}}(490) E \phi_{\mathrm{A}}(570)+\varepsilon_{\mathrm{A}}(490) \phi_{\mathrm{A}}(570)$,
$C_{\mathrm{D}}=\varepsilon_{\mathrm{D}}(490)(1-E) \phi_{\mathrm{D}}(512)$

where $\varepsilon_{\mathrm{D}}(490), \varepsilon_{\mathrm{A}}(490), \phi_{\mathrm{D}}(512)$, and $\phi_{\mathrm{A}}(570)$ are the absorption coefficient at $490 \mathrm{~nm}$ of the donor, the absorption coefficient at $490 \mathrm{~nm}$ of the acceptor, the fluorescence quantum yield at $512 \mathrm{~nm}$ of the donor, and the fluorescence quantum yield of the acceptor at $570 \mathrm{~nm}$ respectively. Consequently, $E$ can be expressed in terms of $R I$ as

$E(R)=\frac{R I(R)-R I(R=\infty)}{R I(R)+R I(R=\infty)\left(\frac{\varepsilon_{\mathrm{D}}(490)}{\varepsilon_{\mathrm{A}}(490)}\right)}$,

where $R I(R=\infty)$ is the ratio of emission peak of the acceptor to that of the donor without FRET, expressed as 
$R I(R=\infty)=\frac{\varepsilon_{\mathrm{A}}(490) \phi_{\mathrm{A}}(570)}{\varepsilon_{\mathrm{D}}(490) \phi_{\mathrm{D}}(512)}$.

\section{Results and discussion}

\section{Purification of double-labeled proteins}

Figure 2(a) shows an example of the purification process of products obtained using the cell-free translation system, monitored by fluorescence detection of the PAGE pattern of protein $33_{\mathrm{D}} 5_{\mathrm{A}}$. The products consist of the properly double-labeled protein; polypeptide with the acceptor, whose translation is stopped at the amber codon (acceptor single-labeled polypeptide); and the short polypeptide without any dye. The sharp strong band around $17 \mathrm{kD}$ corresponds to the double-labeled protein. There are other bands that were not identified, but might have originated from truncated double-labeled polypeptides. The strong background of the gel front consists of aminoacyl tRNA with BODIPY phenylalanine and liberated BODIPY phenylalanine. Both the unexpected bands and strong background appeared in the supernatant of Ni-beads. The final eluted sample contained only the double-labeled full-length

Fig. 2 a Gel images of the purification process of $33_{\mathrm{D}} 5_{\mathrm{A}}$. Lanes are as follows: 1 and 2, cellfree translation solution; 3 and 4 , supernatant of Ni-beads; 5 and 6 , cleaning solution of Ni-beads; 7 , elution of Ni-beads; 8 , final elution by gel filtration chromatography; 9 , fluorescent marker. Top, fluorescence image of $515 \mathrm{~nm}$ emission at $460 \mathrm{~nm}$ excitation; bottom, fluorescence image of $605 \mathrm{~nm}$ emission at $460 \mathrm{~nm}$ excitation. Images were taken on an LAS4000 (GE Healthcare). b Gel images of eight purified samples. Top,

fluorescence image of $520 \mathrm{~nm}$ emission at $488 \mathrm{~nm}$ excitation; center, fluorescence image of $580 \mathrm{~nm}$ emission at $532 \mathrm{~nm}$ excitation. Images were taken on an FMBIO-III (Hitachi Software Engineering). Bottom, western blot analysis of double-labeled His-tagged SNase. Proteins were visualized with an anti-His-tag antibody
SNase. Further purification was carried out by gel-filtration column chromatography. The eight double-labeled proteins were successfully purified, as shown in Fig. 2(b).

\section{Fluorescence spectra}

Fluorescence measurements to evaluate the ratio of the fluorescence intensity from the acceptor to the donor, were repeated several times to assess the degradation of the chromophores. The fluorescence intensity gradually decreased with the number of measurements; the decrease in fluorescence intensity was smaller in $6 \mathrm{M}$ urea than under physiological conditions. The intensity decreased by $\sim 10 \%$ after five measurements: the spectral shape changed very little over the course of the first three measurements, but began to change slightly starting at the fourth measurement. Based on this observation, we concluded that the degradation of the chromophores was negligible for up to three measurements. Consequently, we used the ratio of the fluorescence intensity $(R I)$ for the purposes of evaluation.

Figure 3 shows fluorescence spectra of eight double-labeled SNases in the presence or absence of $6 \mathrm{M}$ urea with excitation at $490 \mathrm{~nm}$. Two peaks are clearly observed in all spectra. The emission peak at $512 \mathrm{~nm}$ is attributed to fluorescence from the a
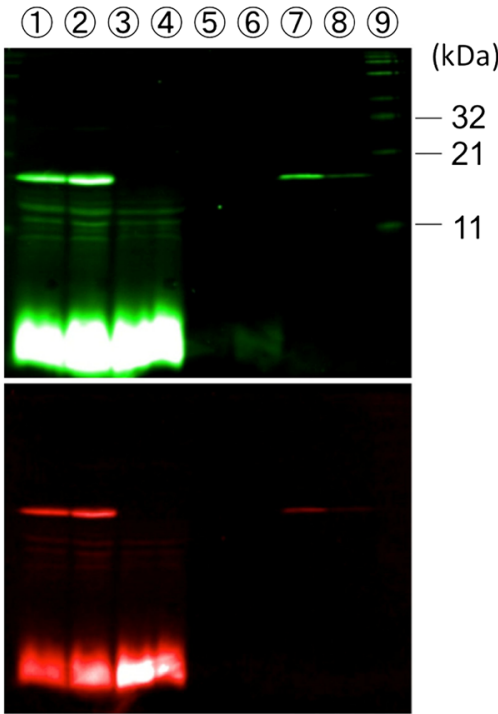

b
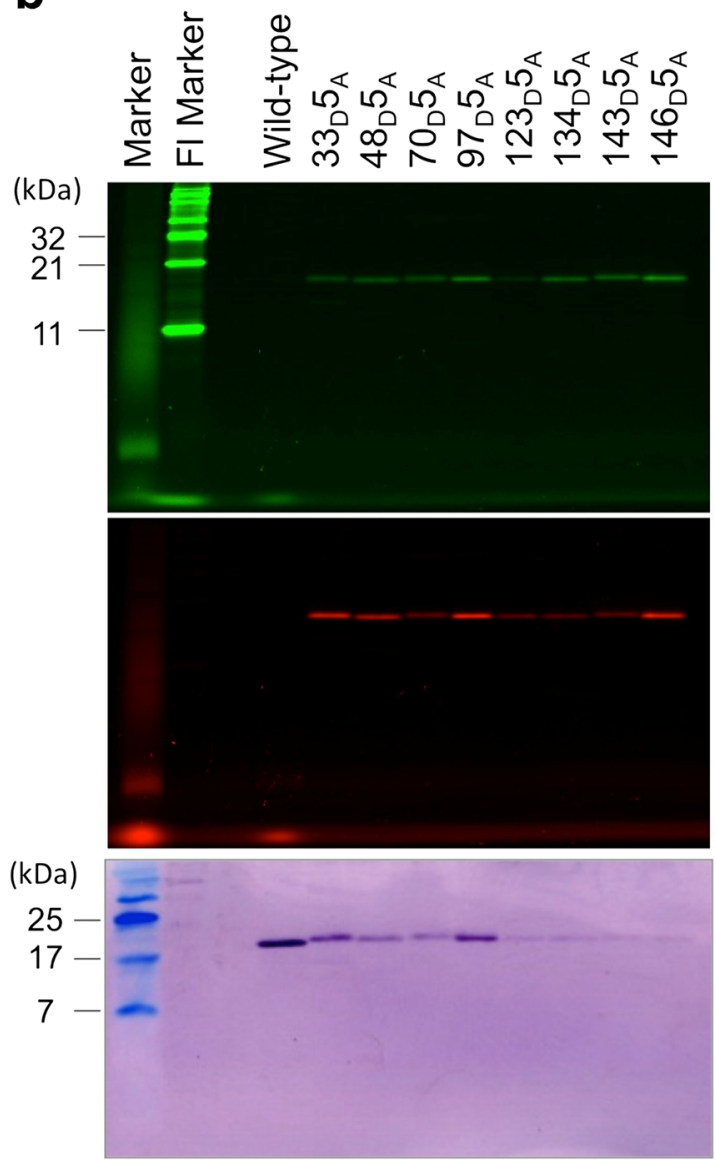
Fig. 3 Normalized fluorescence spectra of double-labeled SNase in the native state (black solid line) and in the presence of $6 \mathrm{M}$ urea (red broken line). a $33_{\mathrm{D}} 5_{\mathrm{A}}, \mathbf{b}$ $48_{\mathrm{D}} 5_{\mathrm{A}}, \mathbf{c} 70_{\mathrm{D}} 5_{\mathrm{A}}, \mathbf{d} 97_{\mathrm{D}} 5_{\mathrm{A}}, \mathbf{e}$ $123_{\mathrm{D}} 5_{\mathrm{A}}, \mathbf{f} 134_{\mathrm{D}} 5_{\mathrm{A}}, \mathbf{g} 143_{\mathrm{D}} 5_{\mathrm{A}}$, and h $146_{\mathrm{D}} 5_{\mathrm{A}}$

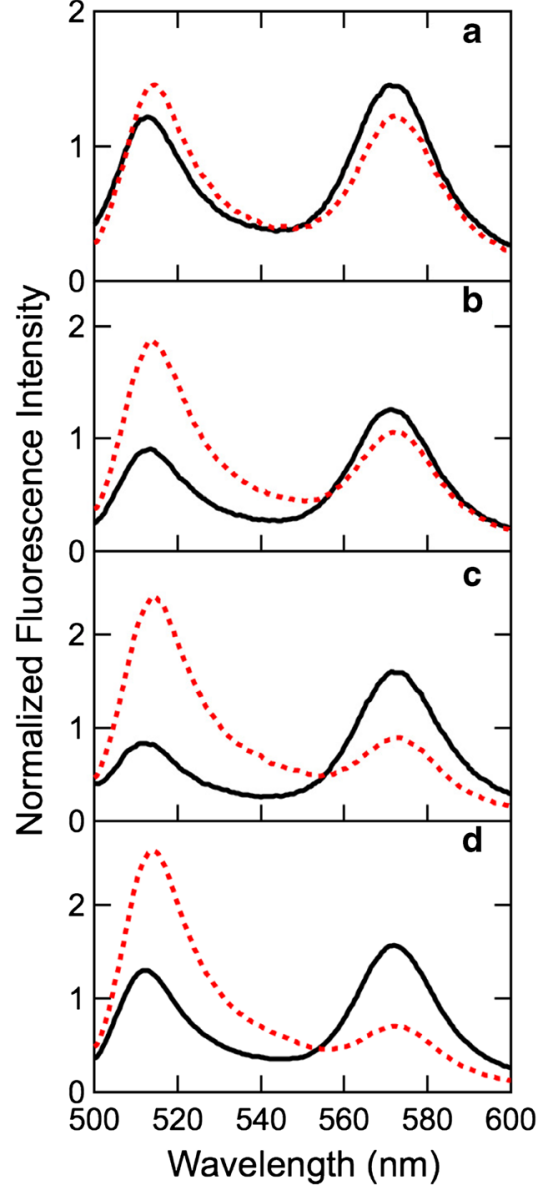

donor, whereas the peak at $570 \mathrm{~nm}$ is attributed to fluorescence from the acceptor. Only the $570 \mathrm{~nm}$ peak was observed when $530 \mathrm{~nm}$ light was used for excitation (data not shown). Because the emission at $570 \mathrm{~nm}$ with $530 \mathrm{~nm}$ excitation results purely from the direct excitation of the acceptor and does not reflect the contribution of FRET, the fluorescence intensity is proportional to the concentration of the acceptor, i.e., the labeled protein. Therefore, we normalized the fluorescence spectra in Fig. 3 against the fluorescence intensity at $570 \mathrm{~nm}$ with $530 \mathrm{~nm}$ excitation so that the normalized fluorescence intensity would be independent of protein concentration. The fluorescence intensity at $570 \mathrm{~nm}$ with $530 \mathrm{~nm}$ excitation did not change upon addition of $6 \mathrm{M}$ urea, which enables us to compare the normalized fluorescence spectra between the presence and absence of urea.

Upon urea denaturation, the emission peak from the acceptor decreased, whereas the peak from the donor increased, indicating that the FRET efficiency of the native state is higher than that of the unfolded state. Thus, the fluorescence spectra are able to probe the structural change due to denaturation. The spectral shapes differed between the labeled sites in both the native and the unfolded states. This site-dependent FRET could provide structural information about the two states.

FRET analyses for native structures were conducted for the fluorescence spectra in Fig. 3. Each spectrum was reconstituted from donor and acceptor fluorescence spectra to obtain $C_{\mathrm{D}}$ and $C_{\mathrm{A}}$ in Eq. (3). Then, $R I$ was obtained from Eq. (4), and $E$ was obtained from Eq. (6). The $R I(R=\infty)$ in Eq. (6) is $0.072 \pm$ 0.001 , which was experimentally obtained by measuring a fluorescence spectrum of a dilute solution of a 1:1 mixture of BODIPY 558 phenylalanine and BODIPY FL phenylalanine. Furthermore, for the native state, the FRET distance between the donor and acceptor $R$ was estimated from Eq. (1). The obtained $R I, E$, and $R$ values are summarized in Table 1 .

\section{Site-dependence of FRET efficiency}

The FRET efficiency $E$ is plotted as a function of the inter-C $\alpha$ distance, $R_{\mathrm{ca}}$, for the native state in Fig. 4 . $R_{\mathrm{ca}}$ was obtained from the crystal structure of SNase (PDB code: 2SNS) (Cotton et al. 1979) and is shown in Table 1. It is difficult to find the $\mathrm{C} \alpha$ atom coordinate of the fifth position in the available crystal structural data because the five $\mathrm{N}$-terminal residues and eight $\mathrm{C}$-terminal residues fluctuate even in the native state. We referred to 2SNS, in which the coordinates of main chain atoms of these residues are specified with a weak electron density distribution.

If $E$ depends only on $R_{\mathrm{ca}}$ as in Eq. (1), $R$ should be proportional to $R_{\mathrm{ca}}$. In fact, $R$ for $48_{\mathrm{D}} 5_{\mathrm{A}}, 70_{\mathrm{D}} 5_{\mathrm{A}}$, and $123_{\mathrm{D}} 5_{\mathrm{A}}$ were 
Table 1 Quantities obtained by FRET analysis

\begin{tabular}{llllllll}
\hline Protein & \multicolumn{1}{l}{ Native state } & & & & \multicolumn{2}{l}{6 M urea } \\
\cline { 2 - 4 } \cline { 7 - 8 } & $R I$ & $E$ & $R(\AA)$ & $R_{\text {ca }}^{*}(\AA)$ & & $R I$ & $E$ \\
\hline $33_{\mathrm{D}} 5_{\mathrm{A}}$ & $1.17(0.02)^{* *}$ & $0.78(0.03)$ & $48(1)$ & 26 & & $0.81(0.01)$ & $0.71(0.04)$ \\
$48_{\mathrm{D}} 5_{\mathrm{A}}$ & $1.38(0.02)$ & $0.81(0.03)$ & $47(1)$ & 42 & & $0.524(0.006)$ & $0.60(0.05)$ \\
$70_{\mathrm{D}} 5_{\mathrm{A}}$ & $1.97(0.02)$ & $0.86(0.02)$ & $44(1)$ & 25 & & $0.326(0.002)$ & $0.46(0.06)$ \\
$97_{\mathrm{D}} 5_{\mathrm{A}}$ & $1.179(0.005)$ & $0.78(0.03)$ & $48(1)$ & 20 & & $0.233(0.005)$ & $0.35(0.06)$ \\
$123_{\mathrm{D}} 5_{\mathrm{A}}$ & $2.23(0.03)$ & $0.88(0.02)$ & $43(1)$ & 20 & & $0.202(0.002)$ & $0.30(0.06)$ \\
$134_{\mathrm{D}} 5_{\mathrm{A}}$ & $1.166(0.006)$ & $0.78(0.03)$ & $48(1)$ & 34 & & $0.177(0.002)$ & $0.26(0.06)$ \\
$143_{\mathrm{D}} 5_{\mathrm{A}}$ & $1.525(0.008)$ & $0.83(0.03)$ & $46(1)$ & $(39)$ & & $0.190(0.002)$ & $0.28(0.06)$ \\
$146_{\mathrm{D}} 5_{\mathrm{A}}$ & $1.141(0.007)$ & $0.78(0.03)$ & $48(1)$ & $(39)$ & & $0.182(0.002)$ & $0.27(0.06)$ \\
\hline
\end{tabular}

$* R_{\mathrm{ca}}$ is given by the distance between $\mathrm{C} \alpha$ atoms. Note that the coordinates of the 143 rd and 146th positions are ambiguous (given by parentheses), as is the fifth position. Because the fifth position is common to all proteins, the values can describe the qualitative relationship

**Estimated errors are given in parentheses following the corresponding values proportional to $R_{\mathrm{ca}}$, as shown in the $R-R_{\text {ca }}$ plot in Fig. 4. However, $R$ for $33_{\mathrm{D}} 5_{\mathrm{A}}, 97_{\mathrm{D}} 5_{\mathrm{A}}$, and $134_{\mathrm{D}} 5_{\mathrm{A}}$ were independent of $R_{\mathrm{ca}}$, indicating that $R$ obtained from $E$ by Eq. (1) is not necessarily reflected in the native structure. Two sets of data, from $143_{\mathrm{D}} 5_{\mathrm{A}}$ and $146_{\mathrm{D}} 5_{\mathrm{A}}$, were excluded from this discussion because these $R_{\mathrm{ca}} \mathrm{s}$ are suspicious from the crystal structure data. Comparison of the position of the donor site between the groups revealed that the former residues are located on long loops, whereas the latter residues are on secondary structural elements. Flexibility of residues may be responsible for these differences in the $R-R_{\mathrm{ca}}$ relationship.

Figure 4 suggests that several factors affect $E$. One of these factors could be the orientation factor $\kappa^{2}$ in Eq. (2), which can have a value between 0 and 4 depending on the orientation (Valeur 2001). In solution, it is usually set at $2 / 3$, under the assumption that the chromophores can take all possible orientations. In the native state, however, the residues are tightly

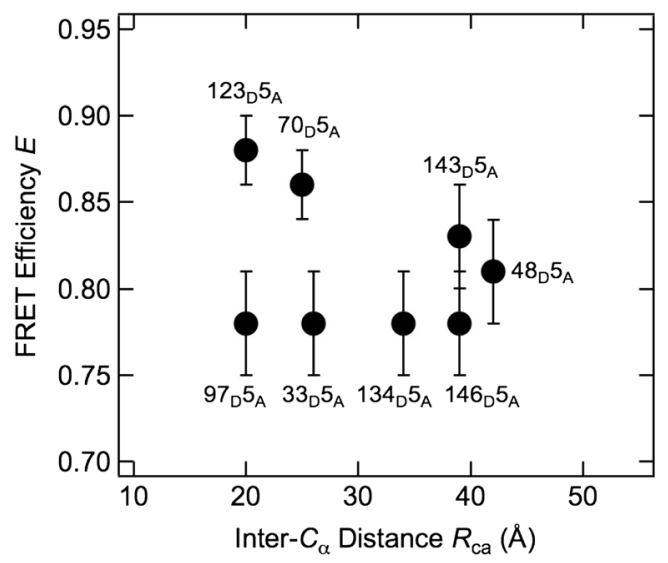

Fig. 4 Comparison of the distance estimated by FRET with the inter-C $\alpha$ distance obtained from the coordinates in 2SNS (Cotton et al. 1979). Note that the coordinates of the 143rd and 146th position are ambiguous. Although the coordinates of the fifth position are also ambiguous, we used as a reference point because this residue is common for all doublelabeled proteins packed and therefore have restricted rotational motion. The effect of the orientation factor could be the origin of the discrepancy between $R_{\mathrm{ca}}$ and $R$ derived from $E$. These observations indicate that labeling at an improper site could yield incorrect structural information.

Figure 5 shows the dependence of $E$ on the inter-residue number, $N$, in the fully unfolded state (i.e., in the presence of $6 \mathrm{M}$ urea). $E$ decreased monotonously as $N$ increased. To describe the statistical properties of the fully unfolded state of SNase, the $E-N$ relationship can be analyzed under an assumption of power-law dependence of $R$ on $N$, as follows (De Gennes 1979):

$R=\alpha N^{\beta}$.

Because the fully unfolded state assumes many conformations, $R$ cannot be determined as a discrete value, but should

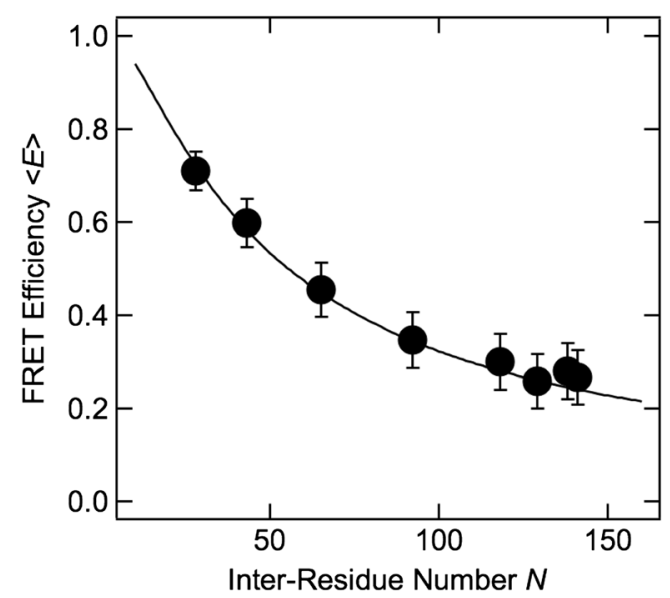

Fig. 5 FRET efficiency of the fully unfolded state in $6 \mathrm{M}$ urea, plotted as a function of inter-residue number. The solid line is the result of fitting, described in the text 
instead be represented by a distribution function. In that case, $E$ is defined by Eq. (9) instead of Eq. (1).

$$
\langle E\rangle=\int p(R) E d R=\int \frac{p(R)}{1+\left(\frac{R}{R_{0}}\right)^{6}} d R,
$$

where $p$ is the distribution of $R$. Here we use the following distribution function,

$p(R)=4 \pi R^{2}\left(\frac{3}{2 \pi\left\langle R^{2}\right\rangle}\right)^{\frac{3}{2}} \exp \left(-\frac{3 R^{2}}{2\left\langle R^{2}\right\rangle}\right)$.

Equation (10) is a three-dimensional Gaussian distribution that is often used in FRET analysis (Sherman and Haran 2006). We assume that the mean distance $R_{\text {mean }}$ obeys the power law in Eq. (8), as follows:

$R_{\text {mean }}=\alpha N^{\beta}$.

The fitting of Eqs. (9)-(11) to the $E-N$ plot in Fig. 5 gives $\beta=0.44 \pm 0.01$. The fitted line is shown in Fig. 5. When the distance distribution is not taken into account, $\beta=0.20 \pm 0.01$ is obtained. Theoretical studies have shown that $\beta=0.588$ for an exclusive-volume random coil in good solvent (Kohn et al. 2004), and experimental studies have consistently shown that $\beta=0.588$ as reflected by measurements of radius of gyration, intrinsic viscosity, etc. for proteins in high-concentration denaturant (Tanford et al. 1966; Kohn et al. 2004). The result obtained in this study, $\beta=0.44 \pm 0.01$, is smaller than previously reported results, although it becomes closer if the distance distribution is taken into account. Note also that the present result refers to a single polypeptide, whereas the previous results were obtained in various proteins. In the other words, the present result is sequence-dependent, whereas previous results were sequence-independent.

Physical quantities such as radius of gyration are probably equivalent to the end-to-end distance of a polymer chain because they all capture the whole molecule. On the other hand, the inter-residue distance probes a segment within a polymer chain. The distribution of a segment could differ from that of a full-length polymer chain, even when they are of the same length, because the conformations of the segment could be restricted by residues outside the segment. Thus, the statistical description of a segment might differ from that of the whole molecule.

The statistical description of various polymer chains could blend all of the unique characteristics together to yield a simple power-law behavior. In fact, $\beta=0.588$ has been obtained even for proteins containing secondary structure (Fitzkee and Rose 2004). On the other hand, in this study, which examined a single molecule, we obtained $\beta=0.44$. The different statistics observed for a single protein may extract information about unique structural features. Although persistent residual structure is not observed in Fig. 5, the fact that $\beta=0.44$ suggests that unfolded SNase has a more compact structure than a random coil. In a previous study, a hydrophobic cluster was observed even in $7 \mathrm{M}$ urea (Neri et al. 1992). Thus, the residue distribution of unfolded SNase might be confined due to hydrophobic collapse. In fact, polymers in poor solvent have $\beta<$ 0.588 ; in this case, the interactions among segments are attractive due to the solvophobic effect. This observation is important and useful for understanding not only the structure of the unfolded state, but also the mechanism of protein folding. It also provides insight into the possible structures of intrinsically disordered proteins.

\section{Conclusion}

We successfully introduced a fluorescent donor and acceptor into SNase using a four-base codon and an amber codon. Using FRET analysis, we carried out a systematic investigation of the distribution of the designated residues in the fully unfolded state. The introduction of artificial amino acids with fluorescent chromophores represents a unique and promising strategy for studying protein folding by FRET. Based on our results, we were able to provide, for the first time, the statistical description of the unfolded structure of a single polypeptide. The data revealed a scaling law distinct from that of a random coil, suggesting that the unfolded structure of SNase is more compact than random coil. On the other hand, distance determination between the donor and the acceptor in the native state is reasonable in some cases but not necessarily in others, depending on the donor position in comparison with the crystal structure. Therefore, the application of FRET analysis to the native state should be performed carefully.

Acknowledgements Authors thank Mr. Yusuke Kishi for his contribution to the early stage of the study. We also thank Ms. Satomi Kayamura for her technical assistance. This work was partly supported by Grants-in-Aid for Scientific Research from the Ministry of Education, Science, Culture, Sports and Technology of Japan to MK (20107006), TH (20107005 and 25102006), and HK (JP25102003).

\section{Compliance with ethical standards}

Conflict of interest All authors declare that they have no conflict of interest

Ethical approval This article does not contain any studies with human participants or animals performed by any of the authors.

Open Access This article is distributed under the terms of the Creative Commons Attribution 4.0 International License (http:// creativecommons.org/licenses/by/4.0/), which permits unrestricted use, distribution, and reproduction in any medium, provided you give appropriate credit to the original author(s) and the source, provide a link to the Creative Commons license, and indicate if changes were made. 


\section{References}

Anfinsen CB (1972) The formation and stabilization of protein structure. Biochem J 128:737-749. https://doi.org/10.1042/bj1280737

Cotton FA, Hazen EE Jr, Legg MJ (1979) Staphylococcal nuclease: proposed mechanism of action based on structure of enzyme-thymidine 3',5'-biphosphate-calcium ion complex at 1.5 - $\AA$ resolution. Proc Natl Acad Sci 76:2551-2555

De Gennes PG (1979) Scaling concepts in polymer physics. Cornell University Press, Ithaca NY

Dill KA (1993) Folding proteins: finding a needle in a haystack. Curr Opin Struct Biol 3:99-103. https://doi.org/10.1016/0959-440X(93) 90208-3

Dill KA, Chan HS (1997) From Levinthal to pathways to funnels. Nat Struct Biol 4:10-19. https://doi.org/10.1038/nsb0197-10

Dill KA, Shortle D (1991) Denatured states of proteins. Annu Rev Biochem 60:795-825. https://doi.org/10.1146/annurev.bi.60. 070191.004051

Fitzkee NC, Rose GD (2004) Reassessing random-coil statistics in unfolded proteins. Proc Natl Acad Sci 101:12497-12502. https://doi. org/10.1073/pnas.0404236101

Hohsaka T, Kajihara D, Ashizuka Y, Murakami H, Sisido M (1999) Efficient incorporation of nonnatural amino acids with large aromatic groups into streptavidin in vitro protein synthesizing systems. J Am Chem Soc 121:34-40. https://doi.org/10.1021/ja9813109

Iijima I, Hohsaka T (2009) Position-specific incorporation of fluorescent non-natural amino acids into maltose-binding protein for detection of ligand binding by FRET and fluorescence quenching. Chembiochem 17:999-1006. https://doi.org/10.1002/cbic. 200800703

Kajihara D, Abe R, Iijima I, Komiyama C, Sisido M, Hohsaka T (2006) FRET analysis of protein conformational change through positionspecific incorporation of fluorescent amino acids. Nat Methods 3: 923-929. https://doi.org/10.1038/nmeth945

Kazmirski SL, Wong KB, Freund SMV, Tan YJ, Fersht AR, Daggett V (2001) Protein folding from a highly disordered denatured state: the folding pathway of chymotrypsin inhibitor 2 at atomic resolution. Proc Natl Acad Sci 98:4349-4354. https://doi.org/10.1073/pnas. 071054398

Kohn JE, Millett IS, Jacob J, Zagrovic B, Dillon TM, Cingel N, Dothager RS, Seifert S, Thiyagarajan P, Sosnick TR, Hasan MZ, Pande VS, Ruczinski I, Doniach S, Plaxco KW (2004) Random-coil behavior and the dimensions of chemically unfolded proteins. Proc Natl Acad Sci 101:12491-12496. https://doi.org/10.1073/pnas.0403643101

Levinthal C (1968) Are there pathways for protein folding? J Chim Phys 85:44 45. https://doi.org/10.1051/jcp/1968650044

Neri D, Billeter M, Wider G, Wüthrich K (1992) NMR determination of residual structure in a urea-denatured protein, the 434-repressor. Science 257:1559-1563. https://doi.org/10.1126/science.1523410

Onuchic JN, Wolynes PG, Luthey-Schulten Z, Socci ND (1995) Toward an outline of the topography of a realistic protein-folding funnel. Proc Natl Acad Sci USA 92:3626-3630. https://doi.org/10.1073/ pnas.92.8.3626

Pappu RV, Srinivasan R, Rose GD (2000) The Flory isolated-pair hypothesis is not valid for polypeptide chains: implications for protein folding. Proc Natl Acad Sci USA 97:12565-12570. https://doi.org/ 10.1073/pnas.97.23.12565

Sherman E, Haran G (2006) Coil-globule transition in the denatured state of a small protein. Proc Natl Acad Sci USA 103:11539-11543. https://doi.org/10.1073/pnas.0601395103

Shortle D, Ackerman MS (2001) Persistence of native-like topology in a denatured protein in $8 \mathrm{M}$ urea. Science 293:487-489. https://doi.org/ 10.1126/science. 1060438

Shortle D, Meeker A (1986) Mutant forms of staphylococcal nuclease with altered patterns of guanidine hydrochloride and urea denaturation. Proteins Struct Funct Genet 1:81-89. https://doi.org/10.1002/ prot.340010113

Tanford C, Kawahara K, Lapanjes SJ (1966) Proteins in 6M Guanidium hydrochloride -demonstration of random coil behavior. J Biol Chem 241:1921-1923

Valeur B (2001) Molecular fluorescence: principles and applications. Weinheim, Wiley-VCH 